\title{
Implantologie \\ Exklusive Fortbildung in Palm Beach Gardens
}

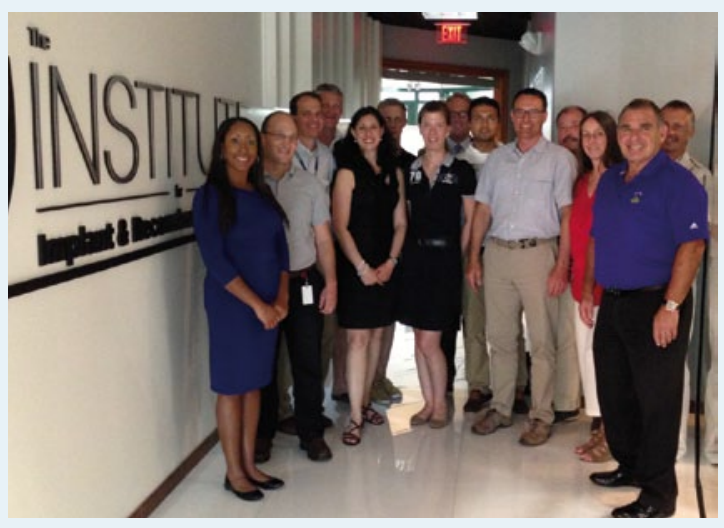

Gastgeber und Teilnehmer der exklusiven Fortbildungsveranstaltung von BIOMET $3 \mathrm{i}$ am Institute for Implant and Reconstructive Dentistry (IIRD) in Palm Beach Gardens, Florida.
BIOMET 3i begrüßte im Juli Zahnärzte aus Deutschland zu einer Fortbildungsveranstaltung im Institute for Implant and Reconstructive Dentistry (IIRD), in Palm Beach Gardens, Florida, USA. Die Teilnehmer beschäftigten sich auf der dreitägigen Veranstaltung mit speziellen Fragestellungen rund um die Implantologie.

Ein ausreichendes Knochenangebot gilt unbestritten als Voraussetzung für die Versorgung mit Implantaten. So war der 1. Trainingspart den verschiedenen Augmentationstechniken gewidmet. Dr. Joachim Schmidt, Direktor der Privatzahnklinik Schloss Schellenstein, vermittelte in seinem Vortrag mit anschließenden praktischen Übungen die Grundlagen der verschiedenen Methoden des Knochenaufbaus anhand eigener Fälle.

Um die implantatreduzierte Versorgung des zahnlosen Kiefers ging es im 2. Block der Veranstaltung. Dr. Robert A. del Castillo, DMD, aus Miami Lakes, beantwortete in seinem praxisbezogenen Vortrag die Frage, wie diese Art der implantatgetragenen Versorgung in der täglichen Praxis umgesetzt und etabliert werden kann. Dabei referierte del Castillo sowohl über die state-of-the-art-Methoden, als auch über neue und künftige Techniken und Technologien in der Implantatprothetik.

Nach einer Pressemitteilung der

BIOMET 3i Deutschland GmbH, Karlsruhe

\section{Transport}

\section{Sichere Aufbewahrung für Prothesen}

Das Aufbewahren von Prothesen ist ein heikles Thema - besonders auf Reisen. Hilfe hierfür schafft die miradent ProthoBox. Die Box gewährleistet eine hygienische Aufbewahrung sowie einen sicheren Transport. Das Innenleben der Box ist in 2 Fächer aufgeteilt. Das große Fach bietet Platz für 2 Prothesen und dient der sicheren Aufbewahrung, auch in einer Reinigungslösung. Damit keine Flüssigkeit auslaufen kann, wird die Dichtigkeit durch eine spezielle Verriegelung gewährleistet. Im kleineren Fach befindet

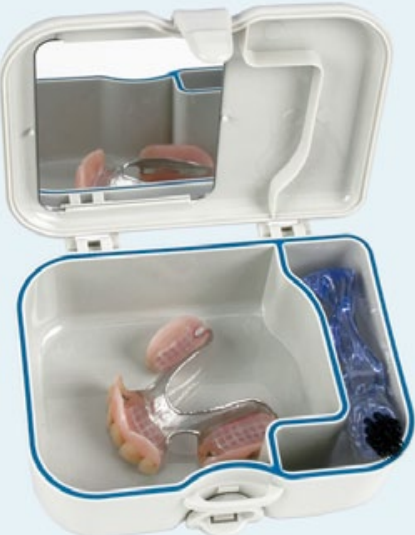

sich eine ergonomisch geformte Reinigungsbürste mit 2 Borstenfeldern. Mit dem weißen Borstenfeld werden Oberflächen gereinigt, mit dem schwarzen die Protheseninnenseiten. Zusätzlich lassen sich in diesem Bürstenfach Reinigungstabletten unterbringen. Ein im Deckel integrierter Spiegel erleichtert dem Prothesenträger das Einsetzen des Zahnersatzes. Die Reinigungsbürste ist auch einzeln erhältlich.

Nach einer Pressemitteilung der

Hager \& Werken GmbH \& Co. KG, Duisburg Internet: www.hagerwerken.de

\section{Propylaxe}

\section{Eine dauerhafte Lösung fürr empfindliche Zähne}

Kuraray Europe führt im September 2013 erstmals einen Desensitizer namens

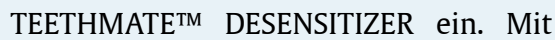
dem Produkt wurde nun ein neues Material ins Leben gerufen, das empfindliche Zähne effektiv behandelt und das erstmalig auf natürliche Weise. Kuraray Noritake Dental hat TEETHMATE DESENSITIZER so entwickelt, dass es das härteste Mineral im Körper, Hydroxylapatit (HAp) bildet. Es entsteht genau dort, wo es benötigt wird, und schließt Dentintubuli und Schmelzrisse. Das neu gebildete HAp verhält sich, als wäre es das des Patienten.
Der Zahnarzt trägt das Produkt lediglich auf einen oder mehrere Zähne auf. Er muss das Pulver mit der Flüssigkeit mischen, auf die Zahnoberfläche auftragen und mit Wasser spülen. Es ist geschmacksneutral und farblos. TEETHMATE DESENSITIZER kann vor und nach dem Bleaching, oder in Kombination mit einem bevorzugten Adhäsiv oder Zement verwendet werden.

Nach einer Pressemitteilung der Kuraray Europe $\mathbf{G m b H}$, Hattersheim am Main Internet: www.kuraraydental.eu

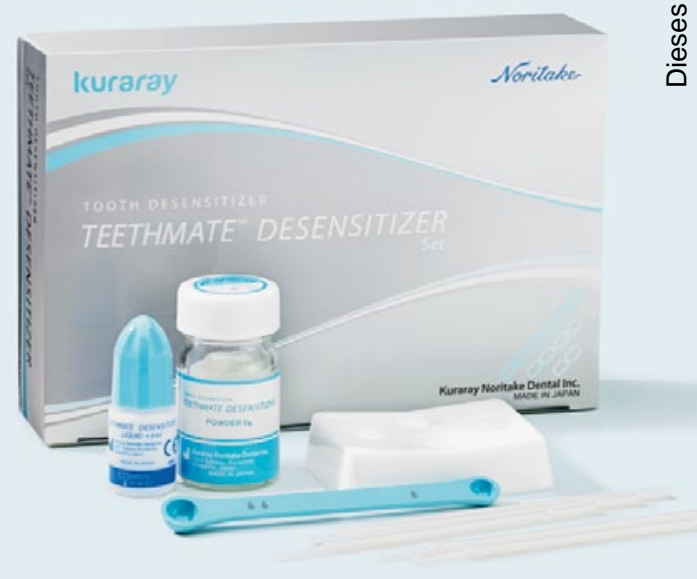




\section{Kristallisieren auf Knopfdruck}

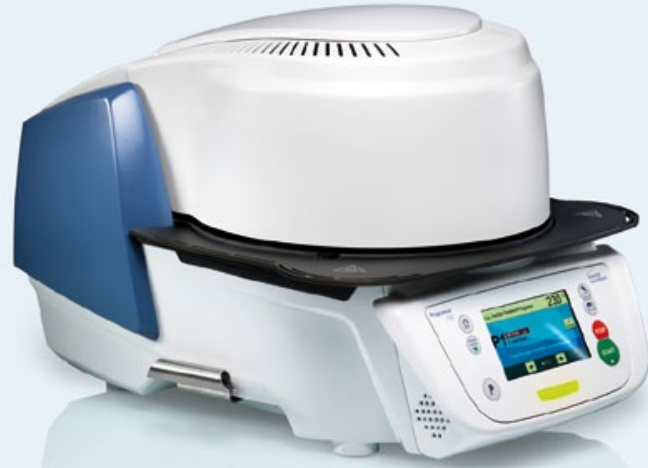

Der Glasur- und Kristallisationsofen Programat CS2 wurde eigens für Zahnärzte entwickelt. Er führt Kristallisations- und Glasurbrände von IPS e.max CAD-Restaurationen einfach und schnell durch. Die 2. Generation des Ofens wird über das neue Farb-Touch-Display und über die bewährte Folientastatur bedient. Der Ofen verfügt über 20 individuell einstellbare Programme und über 7 voreingestellte Ivoclar Vivadent-Programme wie beispielsweise das Speed-Programm. Mit diesem Programm können Einzelzahnrestaurationen in weniger als $15 \mathrm{~min}$ kristallisiert werden. Die Funktion „Automatische Doppelbereich-Temperaturkalibration“ (ATK2) des Programat CS2 sorgt für eine hohe Brenngenauigkeit, indem sie eine vollautomatische Kalibration an 2 unterschiedlichen Temperaturpunkten erstellt. Darüber hinaus ist der Ofen mit der Doppelventil-Vakuumtechnologie versehen. Diese Technologie reduziert die Geräusche der Vakuumpumpensteuerung. Über die Vakuumpumpe wird die Feuchtigkeit im Vakuumschlauch und der Brennkammer nach außen transportiert. Schließlich ist der Ofen mit LAN- und USB-Anschlüssen ausgestattet. Im Servicefall kann der Zahnarzt am Ofen ein Diagnosefile erstellen und dem ServiceCenter per E-Mail weiterleiten.

Nach einer Pressemitteilung der Ivoclar Vivadent $\mathbf{G m b H}$, Ellwangen, Jagst Internet: www.ivoclarvivadent.de

\section{Pilotstudie beståtigt Nutzen von prepCheck}

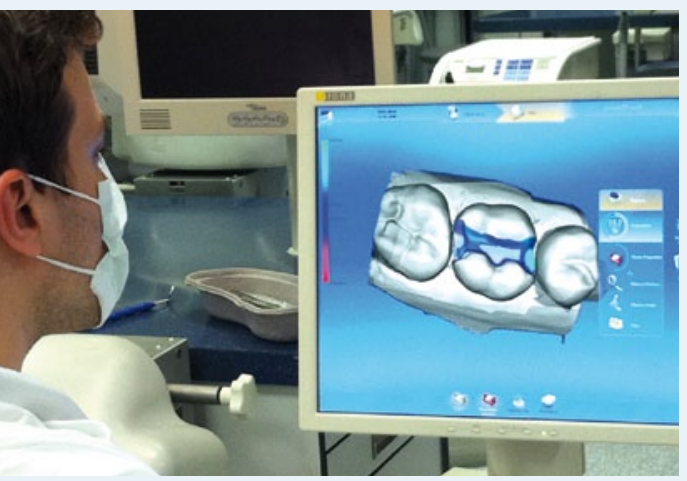

Zahnmedizinstudenten der Universität Frankfurt haben das Anfang des Jahres eingeführte Analysesystem prepCheck getestet. Das System ist leicht zu bedienen und erhöht den Lerneffekt, so das Ergebnis der Evaluierung. Das ergab eine Pilotstudie des Zentrums für Zahn-, Mund- und Kieferheilkunde der GoetheUniversität Frankfurt am Main.

Die Studenten bewerteten das computergestützte Analysesystem prepCheck von Sirona mit der Schulnote „gut“ oder besser. Es dient den Zahnmedizinstudenten dazu, ihre Präparationen zu überprüfen.
Das Tool liefert einheitliche und reproduzierbare Messwerte, die es farbig darstellt. Weitere Ergebnisse aus der Studie: Die Studierenden konnten scharfe Kanten und Undercuts besser erkennen und fanden es hilfreich, die geplante Restauration im Vorfeld beurteilen zu können sowie die eigene Arbeit zu kontrollieren. Darüber hinaus empfehlen die Studierenden, das Analysesystem im Phantomkurs des zahnmedizinischen Studiums sowie bei der Prüfungsvorbereitung einzusetzen. Die Arbeit mit dem System bezeichneten alle Teilnehmer als „einfach“. Seit dem Sommersemester 2013 ist prepCheck im Curriculum des Zahnmedizinstudiums an der Universität Frankfurt integriert. „Die Studierenden arbeiten im Phantomkurs der Zahnerhaltungskunde im 1. klinischen Semester mit dem System. Das Interesse ist groß und das Feedback überaus positiv“, sagt Paul Weigl, Leiter der postgradualen Ausbildung an der Goethe-Universität.

Nach einer Pressemitteilung der Sirona Dental GmbH, A - Wals bei Salzburg Internet: www.sirona.de

\section{Probiotische Therapie beginnt im Mund}

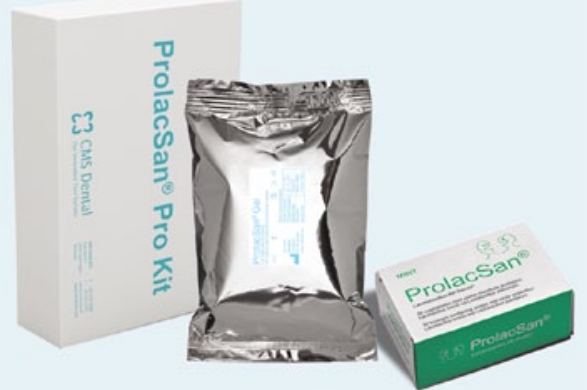

Loser \& Co stellt mit ProlacSan eine wesentliche Ergänzung und Neuheit für die parodontale Therapie vor. ProlacSan ist ein orales Probiotikum. Erstmals ist es möglich, das Wachstum von positiven Bakterien direkt in der Mundhöhle nachhaltig zu fördern und das bakterielle Gleichgewicht länger zu erhalten.

Die Wirkungsweise der beiden ProlacSan ${ }^{\circledR}$ Spezies Lactobazillus brevis 7480 CECT und Lactobazillus plantarum 7481 CECT ist sehr effektiv: Sie unterdrücken das Wachstum pathogener Bakterien und stoßen bakterizide Substanzen aus. Damit beeinflussen sie den Entzündungsprozess. Ihre Charakteristiken wie z.B. die Fähigkeit zur Kolonisierung oder ihre Haftfähigkeit sowie die hohe Resistenz gegen Zahnpasta und Mundspülungen, bewirken einen positiven systemischen Effekt bei lokalen Therapien. Die probiotische Therapie mit ProlacSan wird über die Applikation eines Gels chairside direkt im Anschluss an die Parodontalbehandlung eingeleitet und mit der Einnahme von Tabletten zu Hause vom Patienten fortgeführt. Diese Kombination ermöglicht einerseits die direkte Wirkung des Probiotikums in der Parodontaltasche und trägt andererseits zum Erhalt des positiven Biofilms in der oralen Kavität bis zum nächsten Kontrolltermin bei. Die Prognose bei der Behandlung von Parodontitis, Periimplantitis und Gingivitis wird wesentlich verbessert. Auch bei Halitosis wird ProlacSan zum wichtigen Bestandteil eines befriedigenden Therapiekonzeptes. Das probiotische Ergänzungsmittel hilft, unkontrolliertes Bakterienwachstum und Mundtrockenheit als wichtige Risikofaktoren für die Entstehung von Mundgeruch nachhaltig zu bekämpfen. Das Produkt ist ab sofort über den Fachhandel zu beziehen.

Nach einer Pressemitteilung der Loser \& Co GmbH, Leverkusen

Internet: www.loser.de 
Für den spürbaren

\section{Kaffee-Genuss}

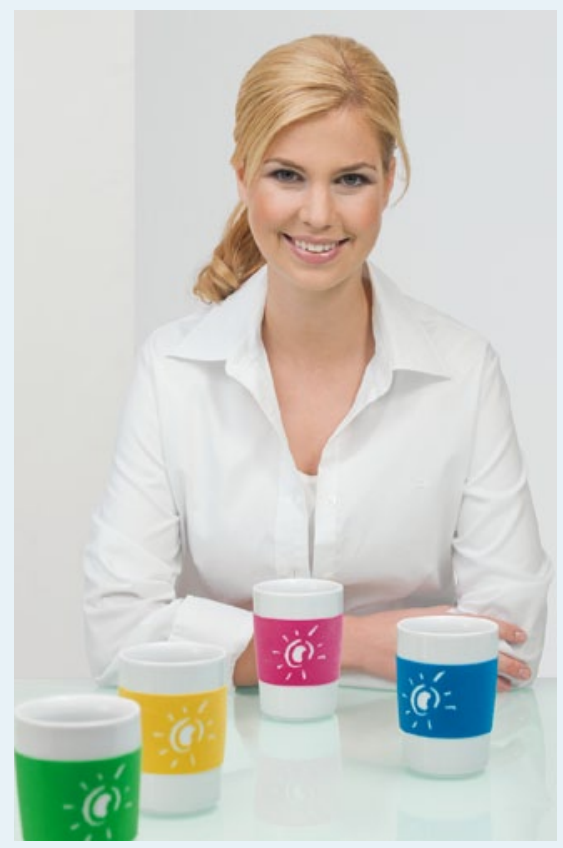

Die Praxis-Collection von Dürr Dental trägt mit edlem Porzellan der Marke Kahla in vielen Praxen zu einem sympathischen Ambiente bei. In diesem Herbst hat sich das Unternehmen für einen ganz besonderen Porzellanartikel entschieden: Maxibecher mit samtweicher Oberfläche aus der Five Senses Touch!-Designreihe von Kahla. Die Präparate der SystemHygiene von Dürr Dental stehen für zuverlässige Desinfektion, Reinigung und Pflege der ganzen Praxis. Sie sind höchst wirksam und zeichnen sich durch sichere Handhabung und angenehmen Duft aus. Die übersichtliche und farbliche Kennzeichnung von Desinfektionsmitteln hat sich seit vielen Jahren in zahllosen Praxen bewährt: Blau für Instrumente, Grün für Flächen, Rosa für Haut und Hände und Gelb für Spezialbereiche. In Anlehnung an dieses logische Farbsystem sind auch die Touch!-Tassen in Blau, Gelb, Rosa und Grün erhältlich.

Vier 2,5-Liter-Flaschen Dürr SystemHygiene und ein 2er-Set Kahla Touch!Tassen können bis 31. Dezember 2013 als Aktions-Paket beim teilnehmenden dentalen Fachhandel bestellt werden. Das Angebot ist gültig in Deutschland und Österreich.

Nach einer Pressemitteilung der DÜRR DENTAL AG, Bietigheim-Bissingen Internet: www.duerr.de

\section{Mundspülung}

\section{Nachhaltige Prophylaxe für Jung und Alt}

Wichtigste Grundlage für eine nachhaltige Mundgesundheit ist die tägliche Dreifachprophylaxe: Dabei werden die mechanische Plaquebeseitigung mittels Zahnbürste sowie die Reinigung der Zahnzwischenräume durch antibakterielle Mundspülungen sinnvoll ergänzt. Die antibakterielle Wirkung basiert auf der speziellen Kombination aktiver Inhaltsstoffe. Die in allen Listerine Mundspülungen enthaltenen lipophilen ätherischen Öle (Eukalyptol, Thymol und Menthol) und das entzündungshemmende Methylsalizylat bekämpfen effektiv den Biofilm und führen nachweislich zu einer deutlichen Reduktion der Keimzahl. Das umfassende Listerine ${ }^{\circledR}$-Sortiment bietet Patienten jeder Altersgruppe die passende Mundspülung:

Mit Listerine Smart Kidz können Kinder ab 6 Jahren anschaulich die Wirksamkeit ei-
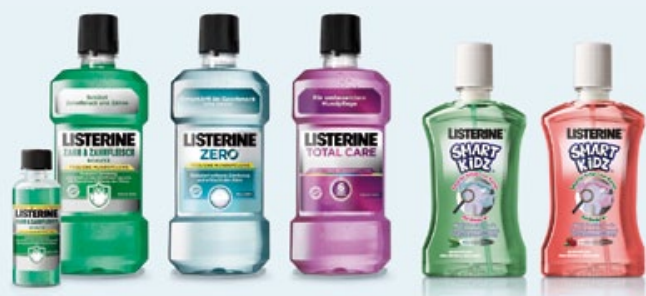

ner Mundspülung aufgezeigt bekommen. Listerine Zero ist milder im Geschmack und enthält keinen Alkohol. Listerine Total Care bietet für Patienten mit Veranlagung zu Karies, Zahnfleischbluten und Zahnsteinbildung eine sinnvolle Unterstützung bei der täglichen Mundhygiene. Für Patienten mit hohem Kariesrisiko oder für Träger von Zahnspangen ist Listerine Zahn- \& Zahnfleisch-Schutz mit Natriumfluorid (100 ppm Fluorid) geeignet.

Nach einer Pressemitteilung der Johnson \& Johnson GmbH, Neuss

\section{Digitales Röntgen \\ Mit den IDS-Highlights auf allen Fachdentals}

Top-Technologie für jede Praxis zugänglich machen - das ist ein zentrales Ziel von Planmeca. „Mit unseren Herbstaktionen werden wir u.a. die DVT-Technologie noch attraktiver machen“, erklärt Dieter Hochmuth, Geschäftsführer der Planmeca Vertriebs GmbH. Planmeca ProMax 3D Plus, das neue Mitglied der ProMaxFamilie, wird auf den Fachdentals mit einem Preisvorteil von ca. $13 \%$ präsentiert. Das Gerät kombiniert DVT, 3D-Gesichtsfoto und 3D-Oberflächenscan mit Panorama- und kephalometrischer Bildgebung sowie extraoralen Bissflügelaufnahmen - bei Volumen von $\varnothing 40 \times 50 \mathrm{~mm}$ bis $\varnothing 140$ x 90 mm. Wer ein DVT-Gerät erwirbt, bekommt kostenlos eine Mitgliedschaft im neuen Planmeca-AnwenderClub. „Wir starten jetzt ein völlig neuartiges Netzwerk. Geboten werden Dialog, Information und Vorteile. Dazu gehören auch Symposien in Finnland, inkl. Werksbesichtigung, Fort- und Weiterbildung“, so Hochmuth. Aufgrund des großen Erfolgs wird das Praxis-NeugründerInnenPaket wieder neu aufgelegt. Damit unterstützt Planmeca, das marktführende Unternehmen im Bereich DVT/Röntgen seine neuen Kunden mit einem ganz be- sonderen Startrabatt. Mehr dazu auch auf den Fachdentals. Die Fachdental-Gäste werden auch die IDS-Neuheiten live erleben können. Darunter der Planmeca ProScanner, ein kompakter KleinbildScanner für Intraoralaufnahmen.

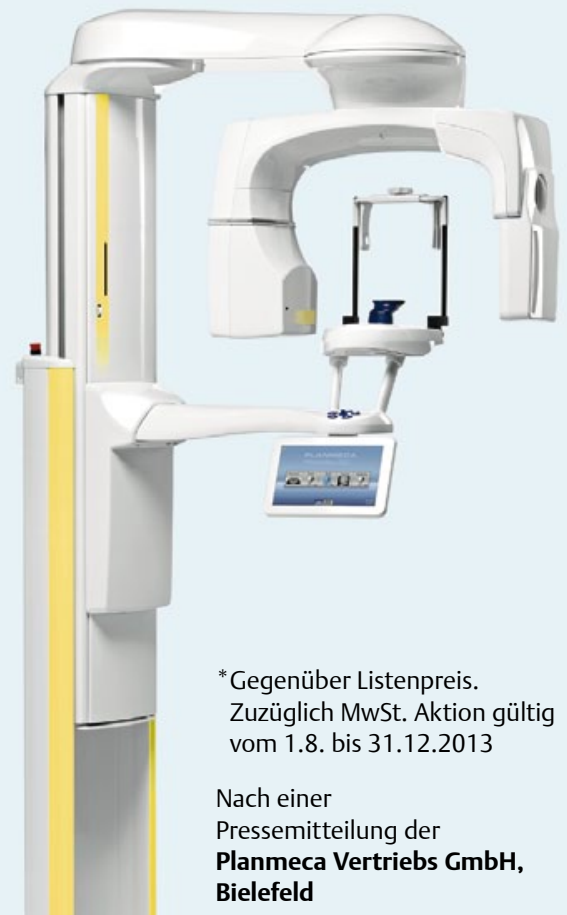




\section{Weltweit wachsendes Interesse am universitåren Nachwuchswettbewerb}

CeramX, das nanokeramische Füllungsmaterial mit dem naturnahen Farbkonzept, spornt auch Zahnmedizinstudenten zu Höchstleistungen an. Der CeramX-CaseContest, der seit 2004 jährlich veranstaltet wird, prämiert herausragende Ergebnisse klinischer Fälle, die von Wettbewerbsteilnehmern aus aller Welt als Step-by-step-Fotodokumentation eingereicht werden. Das Farbkonzept des universell einsetzbaren nanokeramischen Komposits ermöglicht eine natürliche Gestaltung direkter Restaurationen mit nur 7 Farben, die das gesamte VITA-Spektrum* abdecken. Die Einschicht-Version CeramX mono+ um-

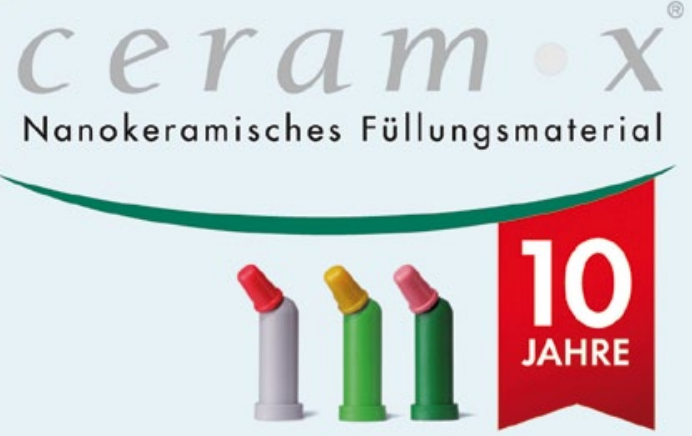

fasst 7 Farben in jeweils einer mittleren Transluzenz. Mit der Zweischicht-Version CeramX duo+ lässt sich die natürlichen Optik von Opazität und Transluzenz besonders genau nachahmen - und dies mit insgesamt nur 4 Dentin-Farben sowie 3 Schmelz-Farben. Im CeramXCase-Contest 2012/2013 haben über
230 Studierende der Zahnmedizin aus 17 Ländern Dokumentationen ihrer klinischen Fälle eingereicht. Führende Kliniker von 90 Universitäten betreuten die Wettbewerbsteilnahme ihrer Studenten als Tutoren. Durch das wachsende Interesse der weltweiten Fachöffentlichkeit entwickelte sich der Case-Contest von CeramX in den letzten 9 Jahren zu einem Highlight der Nachwuchsförderung in der direkten Restaurationstechnik.

Weitere Informationen zu Hands-onFortbildungskursen unter www.dentsply. de/CCC bzw. www.dentsply.de/CeramX.

* VITA ist eine eingetragene Handelsmarke der VITA Zahnfabrik Rauter GmbH\&Co. KG.

Nach einer Pressemitteilung der

DENTSPLY DETREY GmbH, Konstanz

\section{ZR-Schleifer}

\section{Favoriten für die Zirkonbearbeitung}

Zirkonoxid $\left(\mathrm{ZrO}_{2}\right)$ ist ein beliebter Werkstoff in der Praxis - aber faktisch sehr schwer zu bearbeiten. Komet unterstützt den Zahnarzt bei dieser Herausforderung mit dem Set 4637, in dem die „best of"-Instrumente aus der Reihe der ZRSchleifer und Zirkonpolierer zusammengestellt wurden: 4 ZR-Schleifer (2 Formen in mittlerer und feiner Körnung) und eine Flammen- und Kelchform aus dem 2-stufigen Zirkonpoliersystem. Die Spe- zialbindung der ZR-Schleifer bindet die Diamantkörner dauerhaft ein. Daraus resultiert eine gegenüber herkömmlichen Diamantinstrumenten erheblich bessere Abtragsleistung und Standzeit. In nur 2 Polierstufen erzielt der Zahnarzt einen natürlich aussehenden Hochglanz.

Nach einer Pressemitteilung der Komet Dental Gebr. Brasseler GmbH \& Co KG, Lemgo

Internet: www.kometdental.de

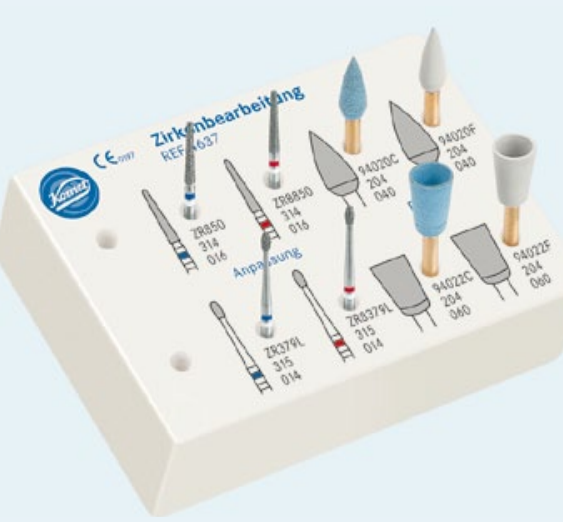

\section{Startschuss für „The only 1“-Kampagne}

Kurz vor ihrem 25-jährigen Firmenjubiläum hat sich die curasan AG selbst ein besonderes Geschenk gemacht und ihr Kernprodukt CERASORB ${ }^{\circledR}$ für den Dentalmarkt zurück erworben. Mit der Kommunikation von Alleinstellungsmerkmalen verleiht curasan dem synthetischen Knochenregenerationsmaterial nun einen längst fälligen Aufmerksamkeitsschub. Hinter den Kernaussagen der, The only 1 “-Kampange verbergen sich belegte und überzeugende Fakten. „Die weltweite Kampagne soll verdeutlichen, dass sich CERASORB ${ }^{\circledR}$ aus der Vielzahl ähnlicher Materialien außerordentlich hervorhebt. Die klinisch dokumentierte Ebenbürtigkeit mit autologem Knochen ist manchen Anwendern bisher vielleicht gar nicht so bewusst“, erläutert Dr. Christine Jäger, Leiterin Vertrieb und Marketing bei der curasan AG. „Das Material ist nicht nur langjährig erfolgreich erprobt, sondern auch bestens geeignet für innovative Applikationsformen “, so
Dr. Jäger. „Schon in Kürze werden wir die beiden jüngsten Neuentwicklungen für den Einsatz bei dentalen Indikationen vorstellen. Dabei werden ein besonders anwenderfreundliches Handling und die Optimierung der Knochenregeneration im Vordergrund stehen." Informationen und Literatur sind auf Anfrage erhältlich.

\section{Nach einer Pressemitteilung der} curasan AG, Kleinostheim

Internet: www.curasan.de 